EVIDENCE REVIEW

\title{
Evidence insufficient to confirm the value of population screening for diabetes and hypertension in low- and middle-income settings
}

\begin{abstract}
S Durão, O Ajumobi, T Kredo, C Naude, N S Levitt, K Steyn, D Bradshaw, T Young
Solange Durão, BSc Dietetics, MPH, is a senior scientist at the South African Cochrane Centre, South African Medical Research Council, Cape Town. Her interests include public health nutrition, evidence synthesis and evidence-informed policies and practice, and capacity building for using and conducting systematic reviews. Oluwayemisi Ajumobi, MS, MPH, is an epidemiologist working at the World Bank, Health, Nutrition and Population Global Practice. Her interests include designing sustainable health innovations, impact evaluations to assess development effectiveness, the impact of health interventions at population level, and strengthening the capacity of the health systems in developing countries for improved multi-disease surveillance. Tamara Kredo, MB ChB, MMed (Clinical Pharmacology), HIV Dip Man, is a senior specialist scientist at the South African Cochrane Centre, Cape Town. Her interests include capacity building in evidence-based healthcare and clinical guideline development and implementation. Celeste Naude, PhD, MNutr, BSc Dietetics, works at the Centre for Evidence-based Health Care, Stellenbosch University, Tygerberg, Cape Town, South Africa. She is a dietitian and senior researcher and her interests include public health nutrition, evidence synthesis, knowledge translation and evidence-informed decision-making. Prof. Naomi (Dinky) Levitt, MB ChB, MD, FCP (SA), Fellow of the University of Cape Town, heads the Division of Diabetic Medicine and Endocrinology, Department of Medicine, UCT and Groote Schuur Hospital and is Director of Chronic Disease Initiative for Africa, Department of Medicine, UCT. Krisela Steyn, MSc, MD, NED, is Associate Director of the Chronic Disease Initiative for Africa, Department of Medicine, UCT. She has conducted research on the public health aspects of non-communicable diseases since 1982. Debbie Bradshaw, DPhil (Oxon), is Director of the Burden of Disease Research Unit, Medical Research Council, Cape Town. She is an expert in disease patterns and has a broad interest in evidence for reducing the burden of disease. Taryn Young, MB ChB, FCPHM, MMed (Public Health) is a public health specialist at the Centre for Evidence-based Health Care at Stellenbosch University and the South African Cochrane Centre. She has considerable experience in building capacity for and promoting the conduct and use of research evidence in healthcare decision-making.
\end{abstract}

Corresponding author: S Durão (solange.durao@mrc.ac.za)

To assess the evidence from systematic reviews on the effect on morbidity and mortality of blanket screening for hypertension or diabetes mellitus compared with targeted, opportunistic or no screening, we searched for relevant systematic reviews and conducted duplicate study selection, data extraction and quality appraisal. Results were summarised narratively. We included two completed reviews of moderate quality and one ongoing Cochrane review. In one completed review, general health checks had no effect on total morbidity or mortality or on healthcare services compared with no health checks. In the other, intensive hypertension screening methods were ineffective in increasing screening uptake or detecting new cases compared with less intensive methods. Both reviews included studies in high-income settings. There is insufficient evidence from currently available systematic reviews to confirm a beneficial effect of blanket screening for hypertension and/or diabetes compared with other types of screening methods in low- and middle-income settings. Scarce resources are being mobilised to implement mass screening intervention for diabetes and hypertension without adequate evidence of its effects. A systematic review is needed to assess clinical effectiveness, cost-effectiveness and overall impact on the health system of screening strategies, especially in low- and middle-income settings such as exist in South Africa. Robust evaluation of these outcomes would then be necessary to inform secondary prevention strategies.

S Afr Med J 2015;105(2):98-102. DOI:10.7196/SAMJ.8819

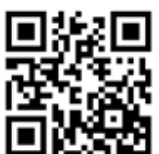

In efforts to address the rising burden of diabetes and hypertension, ${ }^{[1-6]}$ some provincial departments of health in South Africa (SA) are implementing population-level screening programmes for cardiovascular risk factors, including diabetes and hypertension. In addition, the National Ministry of Health included screening for diabetes and hypertension as part of the HIV counselling and testing campaign that commenced in April 2010. Screening seeks to identify apparently healthy individuals who have, or are at increased risk of, cardiovascular disease but do not yet manifest symptoms, with the aim of instituting treatment and impacting favourably on the disease course. ${ }^{[7]}$

Although screening for diabetes and hypertension has the potential to improve health, its effectiveness depends on whether blanket screening (population-wide screening for every individual, irrespective of gender, age group and health conditions), targeted screening (screening targeted at individuals known to be at increased risk for certain conditions/diseases), or opportunistic screening (screening provided at point of care for other related conditions) is

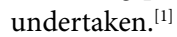

Population-wide screening programmes are associated with high implementation costs, wastage of resources due to ineffective programmes, and inconveniences and psychological distress resulting from false-positive results. ${ }^{[7]}$ To ensure implementation of screening interventions with population-wide benefits, and that are clinically effective and cost-effective, any screening method requires evidence of its effectiveness. ${ }^{[7,8]}$

Since systematic reviews of randomised controlled trials are the gold standard of evidence for the effectiveness of healthcare interventions, we analysed current systematic reviews that have 
evaluated the effects of blanket (population-wide) screening for diabetes and hypertension, compared with targeted or opportunistic screening, or no screening. In this context, it bears noting that systematic reviews use explicit, systematic methods to collate all existing studies that meet prespecified eligibility criteria, and assess the validity of the findings in included studies, to address a clearly stated objective or research question. ${ }^{[9]}$

\section{Methods \\ Criteria for study selection}

We included systematic reviews of population screening (also called blanket or community-wide screening) for diabetes mellitus and/ or hypertension, using any type or combination of screening tests implemented in the general healthy population. These interventions could have been compared with targeted, opportunistic or no screening.

Our primary outcomes included all-cause mortality and diabetesand hypertension-related morbidity. Our secondary outcomes of interest included uptake of the screening, incident cases of hypertension and correctly diagnosed diabetes, impact on the healthcare services, cost-effectiveness and quality of life.

\section{Searching for systematic reviews}

We searched the electronic databases MEDLINE (PubMed), the Cochrane Library, the TRIP database, McMaster Health Evidence. org, the EPPI library and the Campbell Library up to August 2013. We used a comprehensive search strategy, without language restrictions, to identify all existing relevant systematic reviews. Reference lists of included studies were also examined for any other relevant reviews.

\section{Study selection, data extraction and analysis}

Two authors (SD and YA) independently screened search results and extracted data from eligible studies. Disagreements were resolved by discussion with a third author (TK). The methodological quality of included reviews was evaluated using AMSTAR, an 11-item validated tool. ${ }^{[10]}$ Each item on AMSTAR is rated as yes (clearly done), no (clearly not done), unclear or not applicable. Each item rated 'yes' was awarded one point, and points were summed to calculate a total score. Total scores of 0 - 4 indicated low quality, 5 - 8 moderate quality, and $9-11$ high quality. ${ }^{[1]}$ Information from the included reviews was summarised narratively.

\section{Results}

The database search retrieved 875 records. Two completed systematic reviews and one ongoing review were included: Krogsboll et al. ${ }^{[12]}$ assessed the benefits and harms of general health checks in adults, compared with no health checks, on morbidity and mortality, and Ebrahim ${ }^{[13]}$ compared the effectiveness of more and less intensive methods of detecting hypertension. Both reviews involved adult participants and were conducted in highincome settings. The third ongoing study involves a protocol of a Cochrane review that plans to assess the effects of screening for type 2 diabetes on reducing morbidity and mortality, compared with no screening. ${ }^{[14]}$ Table 1 summarises the characteristics of these three reviews.

The Krogsboll and Ebrahim reviews were of high and moderate quality (AMSTAR scores $9 / 11$ and $7 / 10$, respectively). In the case of Ebrahim, it was unclear whether unpublished as well as published studies had been searched for. Neither review reported on publication bias, or assessed possible conflict of interest in the studies they included.

\section{Effects of interventions}

Ebrahim $^{[13]}$ did not address screening for diabetes. Krogsboll et al. ${ }^{[12]}$ assessed interventions that screened for many diseases, including diabetes and hypertension. Table 2 summarises the results of both these reviews.

\section{Effects on mortality and morbidity}

Krogsboll et al. ${ }^{[12]}$ found no effects of general health checks on mortality, either total (risk ratio (RR) $0.99,95 \%$ confidence interval (CI) 0.95 - 1.03; meta-analysis of nine studies, 155899 participants, $\mathrm{I}^{2}=0 \%$ ) or cardiovascular (RR 1.03, 95\% CI 0.91 - 1.17; meta-analysis of eight studies on 152435 participants, $\mathrm{I}^{2}=64 \%$ ). The duration of follow-up was 4 - 22 years, varying within each trial for different outcomes. Health checks compared with no health checks had no effect on morbidity (coronary heart disease, stroke or myocardial infarction).

Ebrahim $^{[13]}$ did not evaluate the effect of screening for hypertension on mortality or morbidity.

\section{Uptake of screening}

Ebrahim $^{[13]}$ found that less rigorous studies, or those conducted in areas with poor healthcare coverage, showed that intensive screening methods (e.g. door-to-door) were more effective at increasing coverage of the population compared with less intensive methods (e.g. routine case finding). However, in well-conducted trials, or in populations with reasonable access to healthcare, this was not the case.

Krogsboll et al..$^{[12]}$ did not assess uptake of screening.

\section{Detection of incident cases of hypertension and diabetes mellitus} Ebrahim $^{[13]}$ found no difference in the yield of unknown hypertensive patients through screening compared with routine case finding. Krogsboll et al.$^{[12]}$ reported that health checks are likely to increase the number of diagnoses, but noted that this outcome was poorly documented in most studies.

\section{Impact on the health service}

Krogsboll et al. ${ }^{[12]}$ found that general health checks had no effect on hospital admission rates, number of people admitted to the hospital once or more, number of days in hospital, or number of physician visits.

\section{Cost-effectiveness and quality of life}

Neither review reported on cost-effectiveness or quality-of-life outcomes.

\section{Discussion}

We found two completed systematic reviews that addressed some aspects of our question regarding population screening v. targeted, opportunistic or no screening for diabetes and hypertension. Krogsboll et al.$^{[12]}$ found that health checks for the general population did not reduce general or cardiovascular-related morbidity and mortality, and results were poorly reported for the effect on new diagnoses and the impact on the healthcare system. Ebrahim ${ }^{[13]}$ found increased coverage with intensive screening in areas with poor healthcare coverage. We also found an ongoing review, the aim of which is to assess the efficacy of screening for type 2 diabetes compared with regular care in reducing morbidity and mortality related to diabetes.

These findings urge caution regarding the implementation of population-based screening interventions. Evidence from a systematic review of economic studies assessing the costeffectiveness of interventions to prevent and control diabetes 
Table 1. Characteristics of included studies

\begin{tabular}{|c|c|c|c|}
\hline & Krogsboll et al..$^{[12]}$ & Ebrahim $^{[13]}$ & Woolthuis et al. ${ }^{[14]}$ (protocol) \\
\hline Objectives of the review & $\begin{array}{l}\text { To quantify the benefits and } \\
\text { harms of general health checks } \\
\text { with an emphasis on patient- } \\
\text { relevant outcomes such as } \\
\text { morbidity and mortality, rather } \\
\text { than on surrogate outcomes } \\
\text { such as blood pressure and } \\
\text { serum cholesterol levels }\end{array}$ & $\begin{array}{l}\text { To determine the most } \\
\text { effective methods of } \\
\text { detecting hypertension, } \\
\text { improving patient adherence } \\
\text { with treatment, improving } \\
\text { control of blood pressure, } \\
\text { and improving professional } \\
\text { compliance with standards } \\
\text { of good practice }\end{array}$ & $\begin{array}{l}\text { To determine the efficacy of screening } \\
\text { for type } 2 \text { diabetes in reducing morbidity } \\
\text { and mortality associated with diabetes } \\
\text { compared with no screening. Secondary } \\
\text { objectives include assessing the effects of } \\
\text { screening for type } 2 \text { diabetes on adverse } \\
\text { events, healthcare consumption, quality of } \\
\text { life, and costs. }\end{array}$ \\
\hline
\end{tabular}

$\begin{array}{ll}\text { Date of last search } & \text { July } 2012 \\ \text { Study designs included } & \text { 14 RCTs } \\ \text { and number of studies } & \\ \text { included } & \end{array}$

Participant characteristics

Setting

Intervention features

Outcomes assessed
Adults, regardless of gender and ethnicity, in the general population or participants within a narrow group (e.g. employees of a company)

High-income countries Primary care or community

General health checks (defined as screening for more than one disease or risk factor in more than one organ system, whether performed only once or repeatedly) compared with no health checks. They included trials that included lifestyle interventions, such as advice on diet, smoking and exercise, in addition to the screening. The interventions could have been administered by doctors, nurses, or other health professionals.

Primary: All-cause mortality, disease-specific mortality (cardiovascular and cancer mortality)

Secondary: Morbidity (myocardial infarction), new diagnosis (total and condition specific), admission to hospital, disability, patient worry, selfreported health, number of referrals to specialists, number of non-scheduled visits to GP, number of additional diagnostic procedures due to positive screening tests, new medications prescribed and frequency and type of surgery, absence from work
July 1996

4 RCTs, 5 quasiexperimental comparisons with contemporary control groups, and 2 before-andafter studies

Age ranged from $\geq 16$ years to adults of all ages.

High-income countries (England, Canada, Wales, Scotland)

Interventions comparing more intensive screening methods for detection of hypertension (e.g. nurse screening, home blood pressure screening, housewife or door-to-door screening) with less intensive methods (e.g. usual care, routine health services check or case-findings, targeted screening, computer doctor prompts)

Coverage of the population achieved and the detection rates of new and known hypertensive patients

\section{N/A}

This review will include only randomised controlled trials with a minimum duration of 3 months.

This review will include people without known diabetes mellitus. The authors will assess the precise diagnostic criteria from the report, or from the authors in case there are missing data.

No specific setting mentioned in the protocol.

This review will include any studies of diabetes screening, including mass, targeted and opportunistic screening approaches, that use the fasting plasma glucose or the 2-hour plasma glucose as a screening test, individually or in combination. This includes studies using stepwise screening procedures, for example questionnaires or database selection, followed by those two tests.

Comparison interventions will be diagnosis of type 2 diabetes during regular care.

Primary: Incidence of type 2 diabetes (as diagnosed at the time of the diagnosis with prevailing diagnostic criteria), diabetesrelated mortality (myocardial infarction, stroke, peripheral vascular disease, renal disease, hyper- or hypoglycaemia or sudden death), total mortality, all morbidity and diabetes and cardiovascular morbidity (vascular complications, neuropathy, retinopathy, nephropathy, erectile dysfunction, amputation)

Secondary: Adverse events, healthcare consumption (e.g. use of medication, number of consultations), quality of life, and costs.

Outcomes will be assessed in the short (3 6 months), medium (6 - 12 months) or long term (>12 months) 
Table 2. Summary of the results of the included completed reviews

\begin{tabular}{|c|c|c|}
\hline & Krogsboll et al. ${ }^{[12]}$ & Ebrahim $^{[13]}$ \\
\hline \multicolumn{3}{|l|}{ Primary outcomes } \\
\hline Effects on mortality & $\begin{array}{l}\text { Total mortality: RR } 0.99 \text { ( } 95 \% \text { CI } 0.95 \text { - 1.03); meta-analysis of } 9 \\
\text { studies, } 155899 \text { participants, } \mathrm{I}^{2}=0 \% \\
\text { Cardiovascular mortality: RR } 1.03 \text { ( } 95 \% \text { CI } 0.91-1.17) \text {; meta- } \\
\left.\text { analysis of } 8 \text { studies on } 152435 \text { participants, } \mathrm{I}^{2}=64 \%\right)\end{array}$ & NR \\
\hline Effects on morbidity & $\begin{array}{l}\text { Health checks had no effect on morbidity in terms of actual } \\
\text { illness, but they may increase the number of people diagnosed } \\
\text { with elevated risk factors. }\end{array}$ & NR \\
\hline \multicolumn{3}{|l|}{ Secondary outcomes } \\
\hline $\begin{array}{l}\text { Effects on uptake/ } \\
\text { coverage of } \\
\text { screening }\end{array}$ & NR & $\begin{array}{l}\text { Results synthesised narratively. More intensive } \\
\text { screening methods (e.g. door-to-door) were more } \\
\text { effective at increasing coverage if studies were } \\
\text { done in areas where coverage was likely to be poor } \\
\text { (e.g. poor black people in US housing blocks) } \\
\text { or with less rigorous BAS. Intensive screening } \\
\text { did not increase coverage greatly in trials that } \\
\text { were well conducted or done in populations with } \\
\text { reasonable access to healthcare. }\end{array}$ \\
\hline $\begin{array}{l}\text { Effect on incident } \\
\text { cases diagnosed }\end{array}$ & $\begin{array}{l}\text { Results synthesised narratively. } \\
\text { General health checks likely to increase the number of new } \\
\text { diagnoses, but these results were poorly reported in most trials. }\end{array}$ & $\begin{array}{l}\text { More intensive screening had no effect on the } \\
\text { detection rates, or yield of unknown hypertensive } \\
\text { patients, compared with routine case finding. }\end{array}$ \\
\hline $\begin{array}{l}\text { Effects on health } \\
\text { services }\end{array}$ & $\begin{array}{l}\text { Health checks had no effects on admission rates, number of } \\
\text { people admitted once or more, or number of days in hospital, } \\
\text { when compared with no health checks. There were also no } \\
\text { effects on physician visits. }\end{array}$ & NR \\
\hline $\begin{array}{l}\text { Process evaluation } \\
\text { data/cost- } \\
\text { effectiveness results }\end{array}$ & NR & NR \\
\hline Quality of life & NR & NR \\
\hline
\end{tabular}

further emphasises this. ${ }^{[15]}$ In this review, three cost-effectiveness studies, one of universal opportunistic screening for undiagnosed type 2 diabetes compared with targeted screening for those with hypertension, and two of universal opportunistic screening for undiagnosed type 2 diabetes with ensuing treatment compared with no screening in a US population aged $\geq 45$ years, showed that universal opportunistic screening was not cost-effective. These cost-effectiveness studies also showed that screening proved cost-effective only when it targeted persons with additional risk factors, such as hypertension, or of specific ethnicities, e.g. African Americans aged 45 - 54 years.

The lack of an effect of general health checks on the healthcare system was surprising, as screening is expected to lead to increased use of healthcare services, to confirm diagnosis and initiate treatment as necessary. Only one trial, conducted in a high-income setting and included in the Krogsboll review, reported an increase in new diagnoses with general health checks compared with no health checks; it is possible that most cases identified in this way were known cases that did not have an additional impact on the health system.

\section{Overall completeness, quality and applicability of evidence}

Neither of the included reviews report on all the specified outcomes. Ebrahim's ${ }^{[13]}$ literature search was conducted in 1996, and is outdated and likely to have missed important new trials addressing the question. The trials included in the reviews were conducted in high-income settings, potentially rendering their results inapplicable to the lower- or middle-income settings that characterise SA. Krogsboll et al. ${ }^{[12]}$ did not assess screening of diabetes and hypertension specifically, but rather screening for multiple diseases or risk factors, including diabetes or hypertension. The results of these interventions may differ from those addressing only hypertension or diabetes.

\section{Conclusion}

Screening interventions should be selected on the basis of evidence of their effects in terms of clinical and healthcare system outcomes. This is particularly important where under-resourced health services are already pressurised to provide care for chronic management of both infectious and non-communicable diseases.

There is insufficient evidence from currently available systematic reviews to confirm a beneficial effect of blanket screening for hypertension and/or diabetes compared with other types of screening methods in low- and middle-income settings. Scarce resources are being mobilised to implement a mass screening intervention for diabetes and hypertension without adequate evidence of its effects.

A systematic review is needed to consider the outcomes of clinical effectiveness, cost-effectiveness and impact on the healthcare system overall of screening strategies, especially in lower- and middle-income 
settings such as exist in SA. Robust evaluation of these outcomes would then be necessary to inform secondary prevention strategies.

Author contributions. All authors contributed to the development of the protocol for this study. SD and YA performed literature searches. SD and YA selected studies for inclusion and TK resolved disagreements in study selection. SD and YA extracted and synthesised data. SD drafted the manuscript. All authors contributed to finalisation of the manuscript, and all reviewed and approved the manuscript.

Acknowledgements. The Cochrane Collaboration's Global Evidence Synthesis Initiative for funding this project.

1. Mohan V, Seedat YK, Pradeepa R. The rising burden of diabetes and hypertension in South East Asian and African regions: Need for effective strategies for prevention and control in primary health care settings. Int J Hypertens 2013;2013: article ID 409083. [http://dx.doi.org/10.1155/2013/409083]

2. Bradshaw D, Norman R, Pieterse D, Levitt NS. Estimating the burden of disease attributable to diabetes in South Africa in 2000. S Afr Med J 2007;97(7):700-706.

3. Norman R, Gaziano T, Laubscher R, et al. Estimating the burden of disease attributable to high blood pressure in South Africa in 2000. S Afr Med J 2007;97(8):692-698.

4. Peer N, Steyn K, Lombard C, Lambert EV, Vythilingum B, Levitt NS. Rising diabetes prevalence among urban-dwelling black South Africans. PLoS One 2012;7(9):e43336. [http://dx.doi.org/10.1371/journal. pone.0043336]
5. Peer N, Steyn K, Lombard C, Gwebushe N, Levitt N. A high burden of hypertension in the urban black population of Cape Town: The Cardiovascular Risk in Black South Africans (CRIBSA) Study. PLoS One 2013;8(11):e78567. [http://dx.doi.org/10.1371/journal.pone.0078567]

6. Mayosi B, Flisher AJ, Lalloo UG, Sitas F, Tollman SM, Bradshaw D. The burden of non-communicable diseases in South Africa. Lancet 2009;374(9693):934-947. [http://dx.doi.org/10.1016/S0140-6736(09)61087-4]

7. Grimes D, Schulz K. Use and abuses of screening tests. Lancet 2002:359(9309):881-884. [http://dx.doi. org/10.1016/S0140-6736(02)07948-5

8. Australian Population Health Development Screening Committee. Population based screening framework Australia: Commonwealth of Australia, 2008. http://www.screening.nhs.uk/criteria (accessed 10 December 2013).

9. Higgins JPT, Green S, eds. Cochrane Handbook for Systematic Reviews of Interventions Version 5.1.0 [update March 2011]. The Cochrane Collaboration, 2011. www.cochrane-handbook.org (accessed 13 December 2013).

10. Shea BJ, Hamela C, Wells GA, et al. AMSTAR is a reliable and valid measurement tool to assess the methodological quality of systematic reviews. J Clin Epidemiol 2009;62(10):1013-1020. [http://dx.doi. org/10.1016/j.jclinepi.2008.10.009]

11. Van der Linde RM, Stephan BCM, Savva GM, Dening T, Brayne C. Systematic reviews on behavioural and psychological symptoms in the older or demented population. Alzheimers Res Ther 2012:4(4):123. [http://dx.doi.org/10.1186/alzrt131]

12. Krogsboll LT, Jorgensen K, Gronhoj L, Gotzsche P. General health checks in adults for reducing morbidity and mortality from disease (Review). Cochrane Database of Systematic Reviews 2013; Issue morbidity and mortality from disease (Review). Cochrane Database of Systematic

13. Ebrahim S. Detection, adherence, and control of hypertension for the prevention of stroke: A

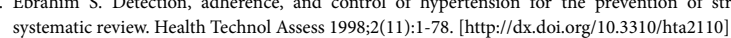

14. Woolthuis EPK, de Grauw WJC, van de Laar FA, Akkermans RP. Screening for type 2 diabetes mellitus (Protocol). Cochrane Database of Systematic Reviews 2005; Issue 2. Art. No.: CD005266. [http:// dx.doi.org/10.1002/14651858.CD005266

15. Li R, Zhang P, Barker LE, Chowdhury FM, Zhang X. Cost-effectiveness of interventions to preven and control diabetes mellitus: A systematic review. Diabetes Care 2010;33(8):1872-1894. [http://dx.do $\operatorname{org} / 10.2337 / \mathrm{dc10}-0843]$

Accepted 25 August 2014 